\title{
Combined liver resection and cytoreductive surgery with HIPEC for metastatic colorectal cancer: Results of a worldwide analysis of 565 patients from the Peritoneal Surface Oncology Group International (PSOGI)
}

\author{
R. Lo Dico a, *, M. Faron ${ }^{\text {b }}$, Y. Yonemura ${ }^{c}$, O. Glehen ${ }^{\text {d, }}$ M. Pocard ${ }^{\text {a }}$, A. Sardi ${ }^{\text {e }}$, M. Hübner ${ }^{\text {, }}$ \\ D. Baratti ${ }^{g}$, G. Liberale ${ }^{\text {h }}$, A. Kartheuser ${ }^{1}$, I. de Hingh ${ }^{j}$, P. Sugarbaker ${ }^{k}$, W. Ceelen ${ }^{\text {, }}$, \\ B. Moran ${ }^{\mathrm{m}}$, M. Robella ${ }^{\mathrm{n}}$, F. Quenet ${ }^{ }$, L. Sideris ${ }^{\mathrm{P}}$, D. Goere ${ }^{\mathrm{b}}$, M. Limbert ${ }^{\mathrm{q}}$, P. Sammartino ${ }^{\mathrm{r}}$, \\ D. Morris s, PSOGI Working Group, BIG RENAPE Working Group
}

\footnotetext{
a Department of Digestive and Oncological Surgery, Lariboisière Hospital, AP-HP, INSERM Unit U1275 / CAP Paris-Tech, Université Sorbonne Paris Cité, Paris, France

b Department of Surgical Oncology and INSERM Unit 1018 CESP Oncostat, Gustave Roussy, Villejuif, France

c Peritoneal Surface Malignancy Center, Kishiwada Tokushukai Hospital, 4-27-1 Kamori-Cho, Kishiwada City, Osaka, 596-8522, Japan

${ }^{\mathrm{d}}$ Department of Surgical Oncology, Hospices Civils de Lyon, Lyon Sud Hospital, Faculty of Medicine, France

e The Institute for Cancer Care, Mercy Medical Center, Baltimore, MD, USA

${ }^{\mathrm{f}}$ Department of Visceral Surgery, Lausanne University Hospital (CHUV), University of Lausanne (UNIL), Switzerland

${ }^{g}$ Peritoneal Surface Malignancies Unit, Fondazione IRCCS Istituto Nazionale dei Tumori, Milan, Italy

${ }^{\mathrm{h}}$ Department of Surgery, Institut Jules Bordet, Université Libre de Bruxelles, Belgium

i Department of Abdominal Surgery and Transplantation, Colorectal Surgery Unit, Saint-Luc University Hospital, Université Catholique de Louvain (UCL), Belgium

j Department of Surgery, Catharina Hospital, P.O. Box 1350, 5602ZA, Eindhoven, Netherlands

${ }^{\mathrm{k}}$ Program in Peritoneal Surface Oncology, MedStar Washington Hospital Center, 106 Irving Street NW, Suite 3900, Washington, DC, 20010, USA

${ }^{1}$ Department of Gastrointestinal Surgery, Ghent University Hospital, Belgium

m Peritoneal Malignancy Institute Basingstoke, Hampshire Hospitals Foundation Trust, Adelmaston Road, Basingstoke, RG24 9NA, UK

${ }^{\mathrm{n}}$ Unit of Surgical Oncology, Candiolo Cancer Institute, IRCCS - FPO, Candiolo, Italy

${ }^{\circ}$ Department of Surgery, Montpellier Cancer Institute, France

$\mathrm{p}$ Department of Surgery, Hospital Maisonneuve-Rosemont, University de Montreal, Quebec, Canada

${ }^{\mathrm{q}}$ IPO, Cirurgia Geral e Proctologia, Hospital Lusíadas Lisboa, Portugal

${ }^{\mathrm{r}}$ Department of Surgery Pietro Valdoni, Sapienza University of Rome, Italy

${ }^{s}$ Department of Surgery, Peritonectomy Center, St George Hospital, Gray St Kogarah, Sydney, NSW, 2217, Australia
}

\section{A R T I C L E I N F O}

Article history:

Accepted 28 July 2020

Available online 26 August 2020

\section{Introduction}

A curative approach in patients with oligometastatic colorectal cancer can achieve "better than expected" long-term survival and provides the rationale for an extensive surgical approach in case of metastases limited in number and to one or a few organs [1].

\footnotetext{
* Corresponding author. Department of Digestive and Oncological Surgery, Lariboisière Hospital, AP-HP, INSERM Unit U1275 / CAP Paris-Tech, Université Sorbonne Paris Cité, 75010, Paris, France.

E-mail address: rea.lo-dico@aphp.fr (R. Lo Dico).
}

Liver metastases (LM) occur in $25-40 \%$ of patients with colorectal cancer (CRC) [2]. The long-term outcomes of patients with LM undergoing systemic chemotherapy alone is poor, with a median overall survival (OS) of 16 months as reported in the Cairo trial [3], and up to 31 months with treatment intensification as shown in the TRIBE trial [4]. A meta-analysis by Franko et al. reported an OS of 19 months in patients with LM from CRC [5]. Curative management of LM is based on surgical resection, although in $70 \%$ of cases, LM will recur despite the use of multimodal and adjuvant chemotherapy [2].

Peritoneal metastases (PM) are present in $3.5-8.3 \%$ of patients 
with CRC at the time of diagnosis [6-8], and are associated with poor survival, being as low as 6 months if left untreated [9]. The presence of isolated PM in patients with CRC is prognostic of poor OS compared with isolated non-peritoneal metastases [5]. Using modern systemic chemotherapy, modest improvements in prognosis can be achieved with median OS in the range of 12-16 months [5,10]. Cytoreductive surgery (CRS) with intraperitoneal chemotherapy, including hyperthermic intraperitoneal chemotherapy (HIPEC) has been proposed as the only potentially curative treatment for PM of CRC origin, achieving a median OS of 31.6 months [11,12]. A median OS up to 41.7 months and a recurrencefree survival of 13.1 months and has also been recently reported in highly selected patients [13]. The National Comprehensive Cancer Network (NCCN) guideline recommends that complete CRS combined with HIPEC can be considered in high-volume centers for selected patients with limited peritoneal metastases in whom R0 resection can be achieved [14].

Simultaneous LM and PM from CRC has traditionally been considered a contraindication to any surgical approach because this disease presentation has been associated with very poor survival $[15,16]$. However, smaller pilot series have reported prolonged survival, reaching up to 3 years in selected patients, after management of simultaneous colorectal LM and PM with CRS plus HIPEC combined with liver resection (LR). The authors claim that LM were not an absolute contraindication to a curative surgical management of PM and the concomitant treatment of both might indeed be possible [17-25]. However, to date, no standard management pathway has been established for patients with simultaneous LM and PM, especially if a major hepatectomy and extensive peritoneal CRS have to be performed. Moreover, there are currently no specific criteria to select patients with the highest potential for surgical success, nor guidelines concerning the timing of peritoneal and liver surgery.

The aim of this study was to assess the early outcomes and survival of CRC patients undergoing LR and peritoneal CRS with HIPEC for concomitant PM and LM. The secondary aim was to identify potential factors related to poorer outcomes, in order to establish a basis to guide the management of these patients, optimizing the selection of candidates for surgical treatment and determining the best sequence of surgical procedures.

\section{Patients and methods}

\section{Data collection}

A prospectively maintained multi-institutional database was established using the PSOGI and BIG-RENAPE database networks from surgical teams of expert centers for colorectal PM performing CRS and HIPEC. This study was carried out in accordance with the precepts established by the Helsinki Declaration and the institutional review board for each center approved the study procedures. Using the databases of 33 international expert centers from 13 different countries, we identified and collected in a retrospective analysis all patients with concomitant PM and LM from CRC treated with LR and CRS with HIPEC between 1993 and 2017.

All background clinical, histological, operative and postoperative data for this study were prospectively collected, entered into a standardized central electronic database and analyzed retrospectively. We included only patients whose clinical records contained complete information such as age, Eastern Cooperative Oncology Group (ECOG) performance status, tumor markers, diagnostic techniques, AJCC stage group (VIII ed.) and histopathology of primary tumor, number and site of LM, surgical procedures used during CRS and LR including complications according to the Clavien-Dindo classification [26], Peritoneal Cancer Index
(PCI) [27], completeness of cytoreduction (CC) score [27], HIPEC techniques and drugs, systemic chemotherapy and eventual druginduced toxicity evaluated according to the National Cancer Institute Common Terminology Criteria for Adverse Events (CTCAE version 4.0) [28] and last complete updated data on follow-up.

Indications for CRS plus HIPEC combined with LR were concomitant metastatic spread to peritoneum and liver from colorectal cancer in patients younger than 75 years of age with adequate cardiac, renal, hepatic and bone marrow function, ECOG performance status 0 to 1 , written informed consent, resectable disease and for the present study patients in whom CRS had a likelihood of achieving residual disease measuring at least $<2.5 \mathrm{~mm}$ (CC1). Contraindications for CRS plus HIPEC combined with LR were extra-abdominal disease, other malignancies, unresectable disease or patients with progressive disease after neoadjuvant chemotherapy and patients whose severe associated medical conditions made them unfit for the procedure. Ovarian metastases were considered a manifestation of peritoneal disease [29].

\section{Operative treatment}

Simultaneous resection was defined as LR and CRS plus HIPEC during the same operation (one step procedure) and separate procedures were defined as two-steps procedures. Detailed staging and evaluation of systemic chemotherapy eventually combined with biological or molecular treatment depended mainly on imaging findings including computed tomography (CT), magnetic resonance imaging (MRI) and positron emission CT (PET-CT). Staging laparoscopy was used mainly for histopathological sampling or when imaging failed to specify resectability. Patients were scheduled for CRS combined with HIPEC and LR according to a likelihood of achieving complete peritoneal cytoreduction (CCO/1) and LR, and dependent on the patient's general condition following discussion at a multidisciplinary meeting including surgeons, medical oncologists, and dedicated radiologists at each PSM center.

At laparotomy, the peritoneal spread was recorded according to the PCI [27]. Patients then underwent surgery with curative intent: CRS of peritoneal disease was performed with peritonectomy and visceral resection according to standardized procedure, albeit some surgical techniques may have varied among centers. Visceral resections and the other peritonectomy procedures were done depending on the distribution of malignancy in the peritoneal space; normal peritoneum was never excised. If macroscopically affected, the appendix and ovaries were systematically removed. HIPEC was administrated after completion of CRS using an open coliseum or closed technique according to the team's preference, to deliver the chemotherapy agent at $42-43^{\circ} \mathrm{C}$ for $30-90 \mathrm{~min}$ in a closed circuit. The drugs employed and the duration of the intraperitoneal chemotherapy was previously described [30,31].

LR was performed according to the principles of oncologic radicality. Minor hepatectomy was defined as any LR of less than three hepatic segments, including atypical resection (metastasectomy, segmentectomy and bisegmentectomy), and radiofrequency ablation (RFA) for lesions measuring less than $2.5 \mathrm{~cm}$ and located far from the main vessels according to each team's preference. Major hepatectomy was defined as the LR of at least three hepatic segments, minor resection was defines as the segmentectomies (anatomical resections) and local ablation was defines as non-anatomical or limited resections (wedge resections, radiofrequency or cryotherapy).

Postoperative complications was defined as taking place within 90 days after surgery and operative mortality as death within 90 days after surgery or until hospital discharge. For 2-staged surgeries we added up the length of stay for both hospital admissions. 


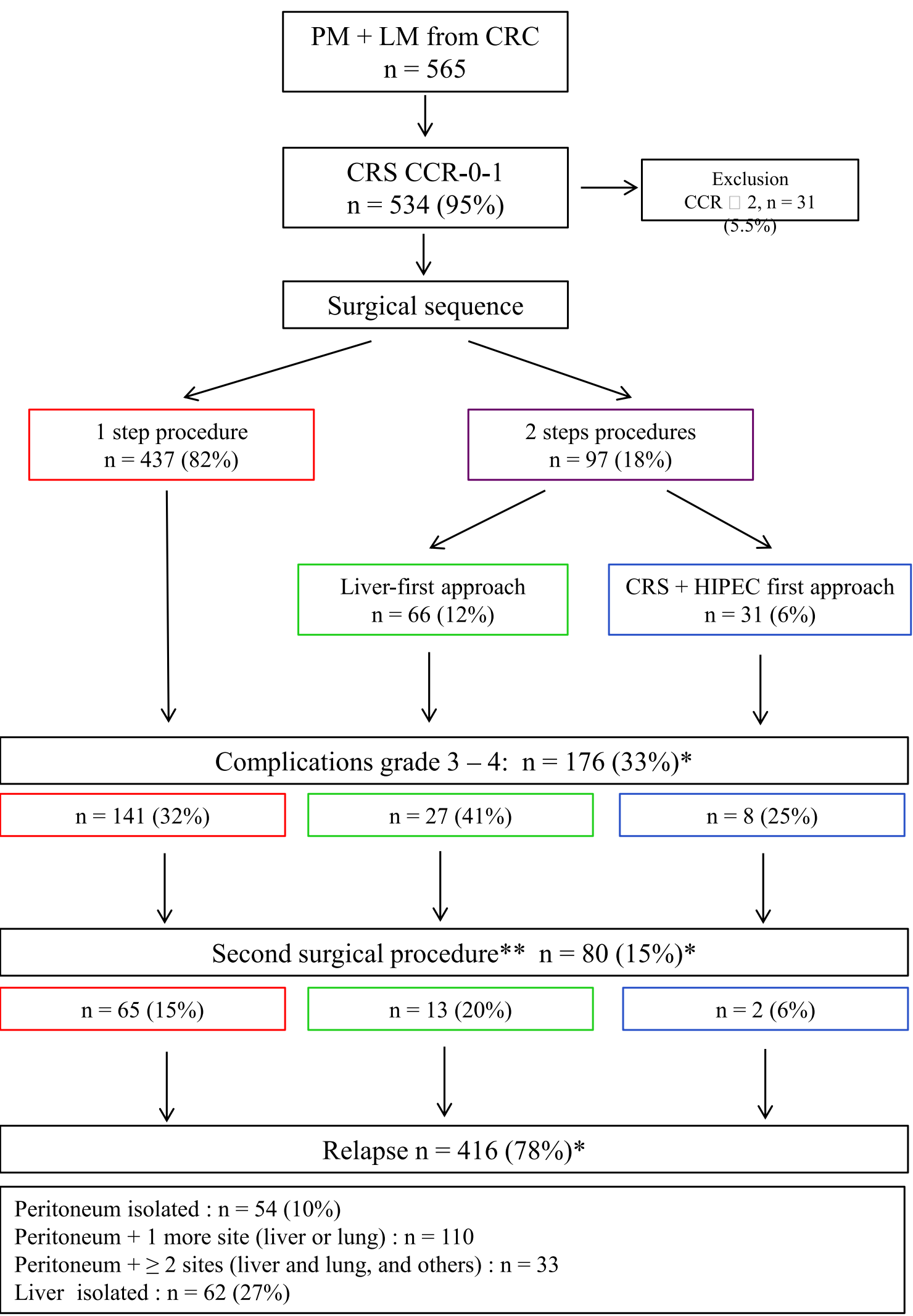

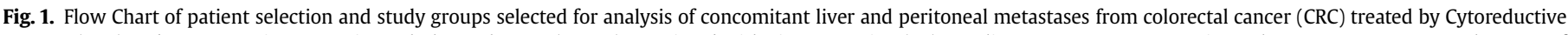

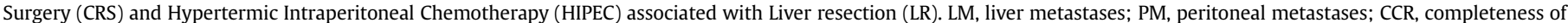
cytoreductive resection; * Data from the global population; **Second surgical procedure for post-operative complications. 
Table 1

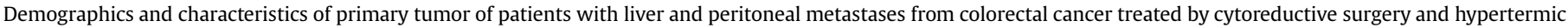
intraperitoneal chemotherapy associated with liver resection.

\begin{tabular}{|c|c|c|c|c|c|}
\hline & ALL & One step & Liver first & HIPEC first & p-value \\
\hline Age & $54.8( \pm 11.7)$ & $54.89( \pm 11.9)$ & $54.6( \pm 10.2)$ & $54.55( \pm 12.1)$ & 0.98 \\
\hline Age $>60$ & & & & & 0.82 \\
\hline No & $329(63.3 \%)$ & 269 (62.7\%) & $41(65.1 \%)$ & 19 (67.9\%) & \\
\hline Yes & $191(36.7 \%)$ & $160(37.3 \%)$ & $22(34.9 \%)$ & $9(32.1 \%)$ & \\
\hline Gender & & & & & 0.3 \\
\hline Female & $312(58.5 \%)$ & $248(57 \%)$ & $44(66.7 \%)$ & $20(62.5 \%)$ & \\
\hline Male & $221(41.5 \%)$ & $187(43 \%)$ & $22(33.3 \%)$ & $12(37.5 \%)$ & \\
\hline Type of Surgery & & & & & 0.17 \\
\hline Laparoscopy & $84(26.7 \%)$ & $59(24.6 \%)$ & $19(37.3 \%)$ & $6(25 \%)$ & \\
\hline Laparotomy & $231(73.3 \%)$ & $181(75.4 \%)$ & $32(62.7 \%)$ & $18(75 \%)$ & \\
\hline Tumor side & & & & & 0.97 \\
\hline Right & 177 (33.9\%) & $142(33.4 \%)$ & $22(33.8 \%)$ & $13(40.6 \%)$ & \\
\hline Left & $271(51.9 \%)$ & $220(51.8 \%)$ & $35(53.8 \%)$ & $16(50 \%)$ & \\
\hline Rectum & $67(12.8 \%)$ & $57(13.4 \%)$ & $7(10.8 \%)$ & $3(9.4 \%)$ & \\
\hline Multifocal & $7(1.3 \%)$ & $6(1.4 \%)$ & $1(1.5 \%)$ & $0(0 \%)$ & \\
\hline T from the TNM & & & & & 0.27 \\
\hline $\mathrm{T} 1$ or $\mathrm{T} 2$ & $14(3.6 \%)$ & $11(3.6 \%)$ & $3(6 \%)$ & $0(0 \%)$ & \\
\hline T3 & $190(49.5 \%)$ & $146(47.2 \%)$ & $29(58 \%)$ & $15(60 \%)$ & \\
\hline $\mathrm{T} 4$ & $180(46.9 \%)$ & $152(49.2 \%)$ & $18(36 \%)$ & $10(40 \%)$ & \\
\hline $\mathrm{N}$ from the TNM & & & & & 0.38 \\
\hline NO & $101(23.3 \%)$ & $76(22.2 \%)$ & $15(25.9 \%)$ & $10(31.2 \%)$ & \\
\hline N1 & 169 (39\%) & $130(37.9 \%)$ & $26(44.8 \%)$ & $13(40.6 \%)$ & \\
\hline $\mathrm{N} 2$ or $\mathrm{N} 3$ & $163(37.6 \%)$ & 137 (39.9\%) & $17(29.3 \%)$ & $9(28.1 \%)$ & \\
\hline Differentiation & & & & & 0.075 \\
\hline Good & 85 (21.7\%) & 65 (21.2\%) & $17(30.4 \%)$ & $3(10.3 \%)$ & \\
\hline Moderate & $225(57.4 \%)$ & $174(56.7 \%)$ & $28(50 \%)$ & $23(79.3 \%)$ & \\
\hline Poor & $82(20.9 \%)$ & $68(22.1 \%)$ & $11(19.6 \%)$ & $3(10.3 \%)$ & \\
\hline Mucinous & & & & & 0.24 \\
\hline No & $295(81 \%)$ & 223 (79.4\%) & 49 (89.1\%) & $23(82.1 \%)$ & \\
\hline Yes & $69(19 \%)$ & $58(20.6 \%)$ & $6(10.9 \%)$ & $5(17.9 \%)$ & \\
\hline Synchronous PM & & & & & 0.0017 \\
\hline No & 317 (60.7\%) & 249 (58.5\%) & $50(76.9 \%)$ & $18(58.1 \%)$ & \\
\hline Yes & $205(39.3 \%)$ & $177(41.5 \%)$ & $15(23.1 \%)$ & $13(41.9 \%)$ & \\
\hline Adjuvant CT & & & & & 0.29 \\
\hline No & $134(25.5 \%)$ & $115(26.9 \%)$ & $12(18.2 \%)$ & $7(21.9 \%)$ & \\
\hline Yes & $392(74.5 \%)$ & $313(73.1 \%)$ & $54(81.8 \%)$ & $25(78.1 \%)$ & \\
\hline
\end{tabular}

TNM: Tumor lymphoNode Metastasis; PM: Peritoneal metastases; CT: Systemic chemotherapy

The general approach across the centers was that patients requiring minor resections had simultaneous LR and peritoneal CRS and HIPEC, whereas in some cases major LR and CRS and HIPEC was performed in two steps: liver-first approach, when the LR was followed by CRS and HIPEC, and delayed LR when the CRS and HPEC was the first procedure.

\section{Endpoints}

The primary endpoint of the analyses was OS. OS was defined as the time from diagnosis of liver or peritoneal metastases to the time of death due to any cause. The secondary endpoints were relapsefree survival (RFS), postoperative morbidity/mortality at 90 days according to the Clavien-Dindo classification [26], and duration of hospital stay. RFS was defined as the time from first surgery, CRS and HIPEC or LR, to relapse, or death, whichever occurred first. RFS at 3 years was defined as the percentage of patients that were relapse-free at three years. In the case of two-steps procedures, the first surgical procedure date was considered as the first treatment day. Second colorectal cancers were considered as RFS events, whereas non-colorectal tumors were disregarded in the analyses.

\section{Statistical analysis}

Quantitative variables were described as mean $(+/-$ standard deviation) or median (inter quartile range) depending on the distribution and compared with Student's t-test or Wilcoxon's test.
Qualitative variables were described as count (percentage) and compared with Chi square test or Fisher's exact test as appropriate.

Survival curves were calculated according to Kaplan-Meier and compared with the log-rank test. Median follow-up was calculated according to Schemper's method [32].

The Cox proportional hazard model was used to determine factors influencing patient prognosis. Any variable achieving a $\mathrm{p}<0.2$ in the univariable analysis was then entered in a Cox multivariable model. Backward variable selection based on Akaike's Information Criteria (AIC) was used to identify the subset of independent variables.

\section{Results}

\section{Patient characteristics}

Five hundred and sixty-five consecutive patients from 33 centers were screened (Fig. 1). Four hundred and ninety-one patients (91.9\%) were treated with preoperative systemic chemotherapy before surgery given to treat PM and/or LM before CRS/HIPEC and LR. The average interval between diagnosis of liver or peritoneal metastases and combined surgery was 6 months. For 224 patients (41.9\%), the PM were synchronous with the primary tumor, for 206 (38.6\%) LM were synchronous with the primary tumor. Regarding the first choice of IP chemotherapy during HIPEC, oxaliplatin (55\%), mitomycin-C (37\%), and "other" (8\%), were reported. Patient characteristics are summarized in Table 1. 
Table 2

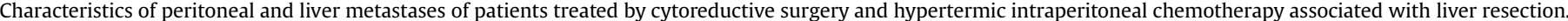

\begin{tabular}{|c|c|c|c|c|c|c|c|c|}
\hline \multirow[b]{2}{*}{ Peritoneal Metastases } & All & \multicolumn{2}{|l|}{ One step } & \multicolumn{2}{|l|}{ Liver first } & \multicolumn{2}{|l|}{ HIPEC first } & \multirow[t]{2}{*}{ p-value } \\
\hline & & & & & & & & \\
\hline CEA pre HIPEC & $\begin{array}{l}28.4( \\
\pm 70.2)\end{array}$ & $24.34( \pm 50.2)$ & & $\begin{array}{l}44.75( \\
\pm 126)\end{array}$ & & $42( \pm 103)$ & & 0.097 \\
\hline CA19.9 pre HIPEC & $\begin{array}{l}79.9( \\
\pm 212.1)\end{array}$ & $88.34( \pm 224)$ & & $\begin{array}{l}30.79( \\
\pm 58.7)\end{array}$ & & $\begin{array}{l}86.51( \\
\pm 272)\end{array}$ & & 0.31 \\
\hline Neoadjuvant CT pre-HIPEC & & & & & & & & 0.013 \\
\hline No & $119(22.8 \%)$ & $86(20.2 \%)$ & & $23(34.8 \%)$ & & $10(32.3 \%)$ & & \\
\hline Yes & $404(77.2 \%)$ & $340(79.8 \%)$ & & $43(65.2 \%)$ & & $21(67.7 \%)$ & & \\
\hline Monoclonal antibodies pre-HIPEC & & & & & & & & 0.87 \\
\hline No & $376(70.3 \%)$ & $305(69.8 \%)$ & & $48(72.7 \%)$ & & $23(71.9 \%)$ & & \\
\hline Yes & $159(29.7 \%)$ & $132(30.2 \%)$ & & $18(27.3 \%)$ & & $9(28.1 \%)$ & & \\
\hline PCI & $9.8( \pm 7.4)$ & $10.07( \pm 7.6)$ & & $\begin{array}{l}9.077( \\
\pm 6.88)\end{array}$ & & $\begin{array}{l}7.552( \\
\pm 4.48)\end{array}$ & & 0.15 \\
\hline PCI $\geq 12$ & $152(30.2 \%)$ & $129(31.5 \%)$ & & $19(29.2 \%)$ & & $4(13.8 \%)$ & & 0.13 \\
\hline CCR & & & & & & & & $<0.0001$ \\
\hline 0 & $472(88.4 \%)$ & 399 (91.3\%) & & $46(69.7 \%)$ & & $27(87.1 \%)$ & & \\
\hline 1 & $62(11.6 \%)$ & $38(8.7 \%)$ & & $20(30.3 \%)$ & & $4(12.9 \%)$ & & \\
\hline Mucinous & & & & & & & & 0.15 \\
\hline No & $304(79.8 \%)$ & 225 (77.9\%) & & $55(88.7 \%)$ & & $24(80 \%)$ & & \\
\hline Yes & $77(20.2 \%)$ & $64(22.1 \%)$ & & $7(11.3 \%)$ & & $6(20 \%)$ & & \\
\hline EPIC & & & & & & & & 0.087 \\
\hline no & $341(91.2 \%)$ & 268 (89.9\%) & & 49 (96.1\%) & & $24(96 \%)$ & & \\
\hline yes & $28(7.5 \%)$ & $27(9.1 \%)$ & & $1(2 \%)$ & & $0(0 \%)$ & & \\
\hline Intra peritoneal oxaliplatin & & & & & & & & 0.14 \\
\hline No & $231(43.2 \%)$ & $180(41.2 \%)$ & & $34(51.5 \%)$ & & $17(53.1 \%)$ & & \\
\hline Yes & $304(56.8 \%)$ & $257(58.8 \%)$ & & $32(48.5 \%)$ & & 15 (46.9\%) & & \\
\hline Adjuvant CT post-HIPEC & & & & & & & & 0.0026 \\
\hline No & $234(44.9 \%)$ & $176(41.5 \%)$ & & $42(63.6 \%)$ & & $16(51.6 \%)$ & & \\
\hline Yes & $287(55.1 \%)$ & $248(58.5 \%)$ & & $24(36.4 \%)$ & & $15(48.4 \%)$ & & \\
\hline Monoclonal antibodies post-HIPEC & & & & & & & & 0.29 \\
\hline No & $461(86.2 \%)$ & $373(85.4 \%)$ & & $61(92.4 \%)$ & & $27(84.4 \%)$ & & \\
\hline Yes & $74(13.8 \%)$ & $64(14.6 \%)$ & & $5(7.6 \%)$ & & $5(15.6 \%)$ & & \\
\hline \multicolumn{9}{|l|}{ Liver Metastases } \\
\hline Number of LM (median [IQR]) & $1[1-2]$ & $1[1-2]$ & $1[1-3]$ & & $2[1-4]$ & & 0.46 & \\
\hline $\mathbf{N}>\mathbf{3}$ & $55(13.1 \%)$ & $33(9.9 \%)$ & $14(23.7 \%)$ & & $8(27.6 \%)$ & & 0.0008 & \\
\hline Liver resection & & & & & & & 0.36 & \\
\hline RO & $454(92.8 \%)$ & $370(92.3 \%)$ & $63(96.9 \%)$ & & $21(91.3 \%)$ & & & \\
\hline $\mathrm{R} 1$ & $35(7.2 \%)$ & $31(7.7 \%)$ & $2(3.1 \%)$ & & $2(8.7 \%)$ & & & \\
\hline Major hepatectomy* & & & & & & & 0.00011 & \\
\hline No & $493(92.3 \%)$ & $413(94.5 \%)$ & $52(81.8 \%)$ & & $28(90.3 \%)$ & & & \\
\hline Yes & $41(7.3 \%)$ & $24(5.2 \%)$ & $14(20.3 \%)$ & & $3(12.5 \%)$ & & & \\
\hline Adjuvant CT & & & & & & & 0.35 & \\
\hline No & $187(38.2 \%)$ & $162(39.5 \%)$ & $17(29.8 \%)$ & & $8(34.8 \%)$ & & & \\
\hline Yes & $303(61.8 \%)$ & $248(60.5 \%)$ & $40(70.2 \%)$ & & $15(65.2 \%)$ & & & \\
\hline
\end{tabular}

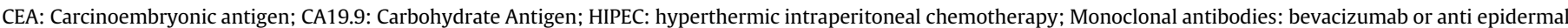

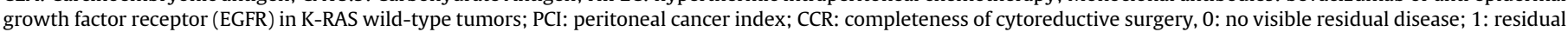

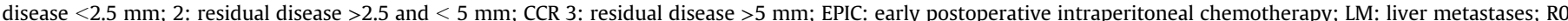

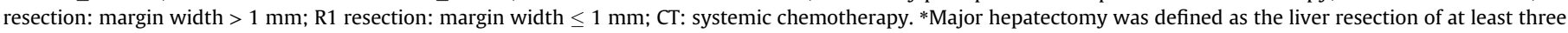
hepatic segments.

\section{Treatment related data}

Median PCI was 9.8 (SD: 7.4, range, 0-39), being $\geq 12$ in 152 patients (Table 2). A complete CRS (CCR-0-1) was achieved in 534 (94.7\%) patients. Four hundred and thirty seven patients $(81.8 \%)$ underwent LR simultaneously with CRS and HIPEC, whereas 97 (18.2\%) had two-steps procedures, among them 66 (12\%) had liverfirst approach (Fig. 1). The median number of LM was 2 (IQR: 1-3, range: $1-14)$. Major LR was performed in 41 patients (7.3\%); fortyfive patients $(8.4 \%)$ were treated with minor resection, and 337 (63.1\%) with local ablation or with the association of two or more limited resections (Table 2).

\section{Early outcome}

The overall severe postoperative complications (grades III-IV) occurred in 176 patients (32.9\%) and surgical interventions for complications were required in 80 patients (15\%). The overall postoperative mortality rate was $4.0 \%(n=23)$. Severe complications occurred in $141 / 437$ (32.3\%) patients who underwent a onestep procedure, and in 35/97 (35.7\%) in two-steps procedures. Briefly: if a liver-first approach was chosen, we observed 6 severe complications after LR (6.1\%) and 21 after CRS and HIPEC surgery (21.6\%); for patients receiving delayed LR we observed 6 complications after CRS and HIPEC surgery (6.1\%) and 2 after LR (2.1\%). The median duration of hospital stay was 24 days (SD: 14.6, range, 5-152 days). Simultaneous CRS and LR was associated with a shorter postoperative hospital stay than two-steps procedures (23 (SD:14.4) vs. 30 (SD: 23.0) days, respectively, $P=0.1$ ) (Table 3). In case of two-steps procedures the durations of the two stays were added to obtain a cumulative stay. The number of LM and the type of LR were not identified as being associated with the frequency of severe postoperative complications (Table 4). 
Table 3

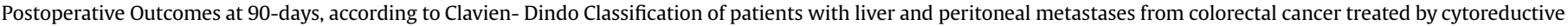
surgery and hypertermic intraperitoneal chemotherapy associated with liver resection.

\begin{tabular}{|c|c|c|c|c|}
\hline \multirow[t]{2}{*}{ Type of Complication } & \multicolumn{2}{|c|}{ Global outcomes } & \multicolumn{2}{|c|}{ Complications by procedures } \\
\hline & No. & $\%$ & CRS + HIPEC & Liver surgery \\
\hline Details of postoperative complications & 317 & $59.4 \%$ & & \\
\hline Hemoperitoneum & 29 & $9.2 \%$ & & \\
\hline Global anastomotic leak & 78 & $24.6 \%$ & & \\
\hline Enteric fistula & 41 & $12.9 \%$ & & \\
\hline Pancreatic fistula & 8 & $2.5 \%$ & & \\
\hline Biliary leak & 10 & $3.2 \%$ & & \\
\hline Urinary fistula & 16 & $5 \%$ & & \\
\hline Other & 3 & $0.9 \%$ & & \\
\hline Intra-abdominal abscess & 42 & $13 \%$ & & \\
\hline Wound infection & 14 & $0.44 \%$ & & \\
\hline Peritonitis & 3 & $0.9 \%$ & & \\
\hline Pleural effusion with drainage & 45 & $14.2 \%$ & & \\
\hline Pneumonia & 16 & $5 \%$ & & \\
\hline Respiratory distress & 5 & $0.3 \%$ & & \\
\hline Pulmonary embolism & 2 & $0.1 \%$ & & \\
\hline Ileus & 5 & $0.3 \%$ & & \\
\hline \multicolumn{5}{|l|}{ Complications (Clavien Dindo)* } \\
\hline 0 & & & $217(40.6 \%)$ & $515(96.4 \%)$ \\
\hline 1 & & & $54(10.1 \%)$ & $8(1.5 \%)$ \\
\hline 2 & & & $87(16.3 \%)$ & $2(0.4 \%)$ \\
\hline 3 & & & $19(3.6 \%)$ & $2(0.4 \%)$ \\
\hline 3a & & & $56(10.5 \%)$ & $3(0.6 \%)$ \\
\hline $3 b$ & & & $45(8.4 \%)$ & $0(0 \%)$ \\
\hline 4 & & & $48(8.9 \%)$ & $3(0.6 \%)$ \\
\hline 5 & & & $8(1.5 \%)$ & $1(0.18 \%)$ \\
\hline 90-day postoperative major complications (grade III-IV) & 176 & $32.9 \%$ & & \\
\hline \multicolumn{5}{|l|}{ Reoperation } \\
\hline No & & & $485(86.5 \%)$ & $61(93.8 \%)$ \\
\hline Yes & & & $76(13.5 \%)$ & $4(6.2 \%)$ \\
\hline Mortality & 23 & $4 \%$ & & \\
\hline \multicolumn{5}{|l|}{90 days mortality } \\
\hline No & & & $539(96.8 \%)$ & $78(94 \%)$ \\
\hline Yes & & & $18(3.2 \%)$ & $5(6 \%)$ \\
\hline Hospital stay (days) & 23 & & $22.9( \pm 14.6)$ & $14.1( \pm 13.5)$ \\
\hline
\end{tabular}

${ }^{*}$ Dindo D, Demartines N, Clavien PA: Classification of surgical complications: a new proposal with evaluation in a cohort of 6336 patients and results of a survey. Ann Surg 240:205-13, 2004.

\section{Long-term outcome}

The median follow-up was 48.4 months (95\% CI 44.2-56.2). The median OS was 47.6 months (95\% CI 42.5-53.3), with 1-, 3- and 5year OS rates being $93 \%, 64 \%$ and $39 \%$ respectively (Fig. 2). The median RFS was 19.4 months (95\% CI, 17.4-20.7), with 1-, 2- and 3year RFS rates being $74 \%, 37 \%$, and $21 \%$ respectively (Fig. 2). Four hundred and sixteen $(77.9 \%)$ patients had a recurrence during the follow-up. The liver was the most frequent site of recurrences $41.1 \%$ $(\mathrm{n}=231)$. Among this group, 62/231 (27\%) had isolated liver recurrence. Fifty-four (10\%) patients had isolated peritoneal recurrence, 213 (39.9\%) patients had extra-peritoneal recurrence, and 143 (26.8\%) patients had both. Among the patients with extraabdominal recurrence, 138 (24.4\%) had pulmonary metastases, either isolated or in combination with other sites. Overall, 282 (49.9\%) patients died during the follow-up period.

The sequence of the surgery had an impact on the survival (Fig. 3A and B). For patients treated with liver-first approach, the median OS and RFS were better than patients treated with CRS and HIPEC first or for patients who had one-step procedure (63.7, 52.6 and 44.8 months respectively for OS $(P=0.036)$, and $28.2,17.1$ and 17.8 for RFS, $(P=0.016)$ ). Upon univariate analysis, $\mathrm{PCI}$, adjuvant LR chemotherapy and surgical sequence, were identified as prognostic factors for lower OS. Moreover, our results have shown a trend towards a lower OS after use of different than oxaliplatin-based drugs for IP compound and modern systemic chemotherapy. At multivariate analysis, only PCI remained as a significant prognostic factor (Table 5). Kaplan-Meier curves for OS of all patients and stratified by surgical sequence are shown (Fig. 3A). At univariate analysis, higher PCI and surgical sequence were both identified as greater prognostic factors for lower RFS than primary tumor side. These results were confirmed on multivariate analysis (Table 6). The Kaplan-Meier curves of all RFS patients, stratified by surgical sequence, are shown in Fig. 3B.

\section{Discussion}

The management of patients with liver and peritoneal metastases from CRC has undergone major improvements over the past few decades. Whereas OS did not exceed one year with classic systemic chemotherapy based on 5-FU [33], oxaliplatin and targeted therapies such as anti-angiogenic or anti-EGFR antibodies (for wild type RAS) have allowed extending the OS up to 2 years in selected patients $[5,20,34]$. Recent studies have suggested that resection of liver and peritoneal metastases, combined with HIPEC, may increase OS up to 3 years, despite an increased risk of morbidity [17,19]. Recently, the combination of three systemic chemotherapy agents (FOLFOXIRI regimen) has shown an increased OS of several months in metastatic CRC patients compared to classical chemotherapy regimens [4], and some achieve OS similar to extensive surgery. However, the incidence of serious adverse events in patients treated with FOLFOXIRI plus bevacizumab is up to $20.4 \%$ [35], which is comparable to perioperative morbidity of major surgery (5-28\% for CRS and HIPEC [16] and 5-20\% for major liver resection) [36-38]. 
Table 4

Univariate analysis of risk factors for complications of patients treated by cytoreductive surgery and hypertermic intraperitoneal chemotherapy associated with liver resection for liver and peritoneal metastases from colorectal cancer.

\begin{tabular}{|c|c|c|}
\hline & OR $[95 \% \mathrm{CI}]$ & $\mathrm{p}$ value \\
\hline Age & $0.99[0.98-1.01]$ & 0.23 \\
\hline Age $>60$ years & & 0.41 \\
\hline No & 1 & \\
\hline Yes & $0.85[0.58-1.24]$ & \\
\hline Treatment after year 2000 * & & 0.3 \\
\hline No & 1 & \\
\hline Yes & $0.68[0.34-1.42]$ & \\
\hline PCI (for each more point) & $1.03[1.01-1.06]$ & 0.0084 \\
\hline $\mathrm{PCI}>12$ & & 0.007 \\
\hline No & 1 & \\
\hline Yes & $1.73[1.16-2.56]$ & \\
\hline EPIC & & 0.36 \\
\hline No & 1 & \\
\hline Yes & $1.21[0.52-2.67]$ & \\
\hline Intraperitoneal Oxaliplatin & & 0.041 \\
\hline No & 1 & \\
\hline Yes & 0.69 [0.48-0.99] & \\
\hline Neoadjuvant CT for PM & & 0.31 \\
\hline No & 1 & \\
\hline Yes & $1.26[0.81-1.98]$ & \\
\hline Number of LM & $1.00[0.88-1.13]$ & 0.98 \\
\hline$>3 \mathrm{LM}$ & & 0.88 \\
\hline No & 1 & \\
\hline Yes & $1.05[0.56-1.9]$ & \\
\hline Major hepatectomy & & 0.77 \\
\hline No & 1 & \\
\hline Yes & $1.11[0.54-2.21]$ & \\
\hline
\end{tabular}

PCI: peritoneal cancer index; EPIC: early postoperative intraperitoneal chemotherapy; CT: systemic chemotherapy; PM: Peritoneal metastases; LM: Liver metastases; Major hepatectomy was defined as the liver resection of at least three hepatic segments. *Treatment after year 2000: the analysis was stratified by time periods (before and after 2000) to discern the effect of modern oxaliplatin-based.

This multicenter study is the largest series of selected patients with PM from CRC and simultaneous LM treated with LR, CRS and HIPEC. The present study shows that extended surgical management of multi-metastatic CRC is feasible with an acceptable morbidity and reasonably low postoperative mortality rates (31\% and $4 \%$, respectively). These morbidity and mortality rates are consistent with those reported after LM resection and similar to PM treatment alone $[13,17,23,34]$. We believe these low rates of morbidity were achieved by careful selection of patients: if LM required only minor $\mathrm{LR}$, this was usually performed at same time as CRS + HIPEC. However, if LM required complex or major LR, especially in patients with suspected liver parenchyma damage through preoperative chemotherapy, resection of LM was mostly performed non-simultaneously to CRS and HIPEC. Interestingly, despite this approach, a major LR was not associated with an increased complication rate compared with minor LR. However, we suggest that this concept of two-steps procedures, already used in complex abdominal and liver surgeries, may represent a valuable tool to reduce patient morbidity and mortality rates. Despite this cautionary note, In light of the present data, it chould be concluded that when both LM and PM are resectable, extended surgery improves chances for selected patients to achieve favorable OS rates. Surprisingly, in this study, we found that for patients treated with liver first approach the median RFS was significantly better than other surgical sequence strategies. In order to further discuss available surgical sequence strategies [39] to be advocated in case of advanced CRC (liver metastases) with asymptomatic primary, a recent meta-analysis [40] demonstrated that no significant differences in long-term survival and major morbidity were found amongst the simultaneous, delayed and liver-first approach. Moreover, the reverse strategy (so-called liver-first approach) was ranked as the potentially best treatment with respect to its relative efficacy on the basis of 5-year OS outcomes and postoperative complication rate compared to simultaneous or delayed LR. Probably because the risk that LM becomes unresectable during the interval between two surgeries is real, some centers have chosen to realize liver-first approach. The better OS and RFS rates between the different strategies showed in this study suggested that the liver-first approach strategy might be an appropriate option for the

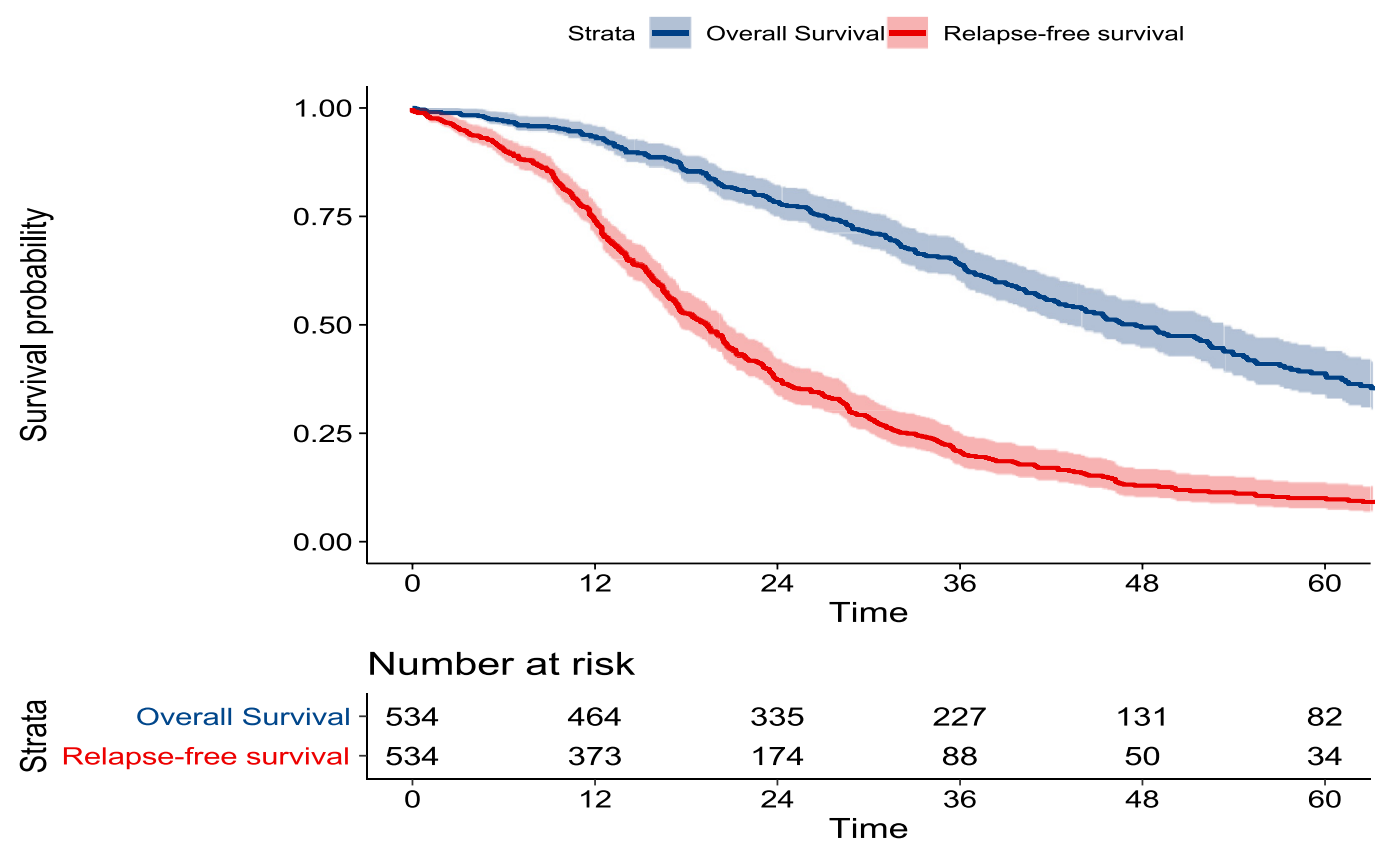

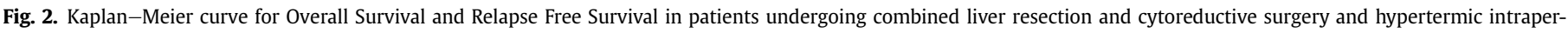
itoneal chemotherapy for concomitant colorectal liver and peritoneal metastases. 
A

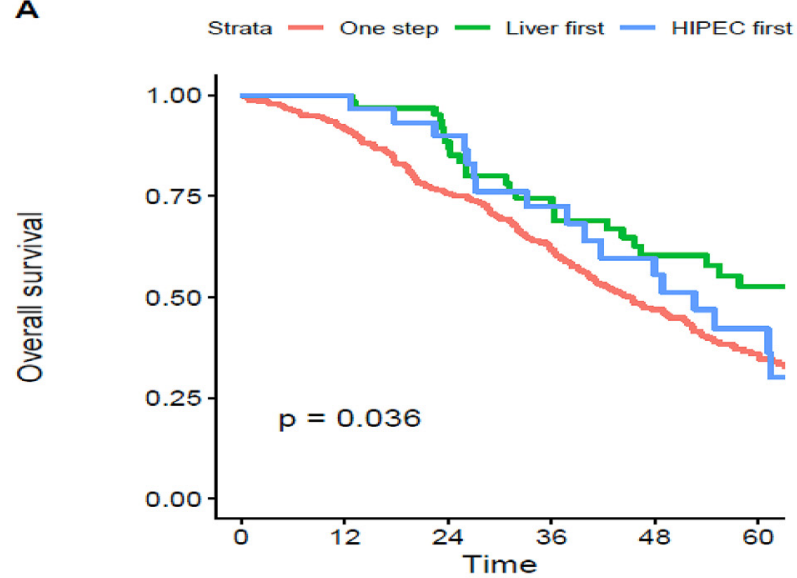

Number at risk
B

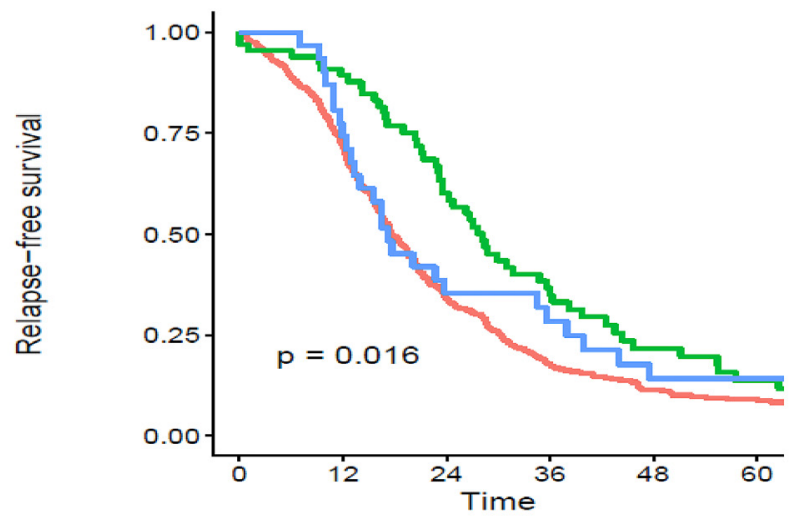

Number at risk
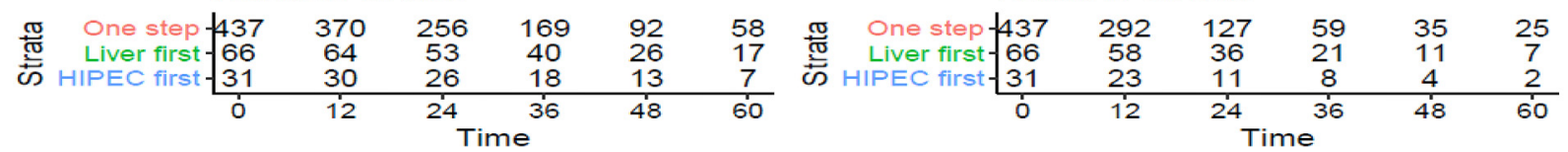

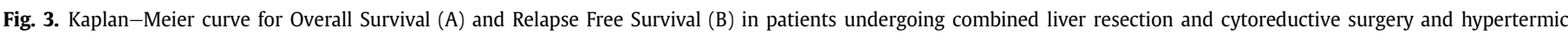

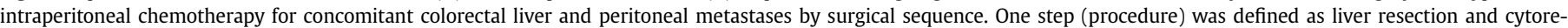

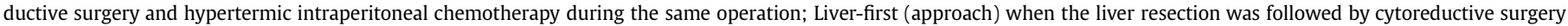
and hypertermic intraperitoneal chemotherapy, HIPEC-first when liver resection was delayed.

Table 5

Uni and multivariable analysis for Overall Survival of patients treated by cytoreductive surgery and hypertermic intraperitoneal chemotherapy associated with liver resection for liver and peritoneal metastases from colorectal cancer.

\begin{tabular}{lll}
\hline & OR [95\% CI] & p value \\
\hline Age & $0.99[0.98-1.01]$ & 0.23 \\
Age $>$ 60 years & 1 & 0.41 \\
No & $0.85[0.58-1.24]$ & \\
Yes & & 0.3 \\
Treatment after year 2000* & 1 & \\
No & $0.68[0.34-1.42]$ & \\
Yes & $1.03[1.01-1.06]$ & 0.0084 \\
PCI (for each more point) & & 0.007 \\
PCI > 12 & 1 & \\
No & $1.73[1.16-2.56]$ & 0.36 \\
Yes & 1 & 0.041 \\
EPIC & $1.21[0.52-2.67]$ & \\
No & & \\
Yes & 1 & 0.31 \\
Intraperitoneal Oxaliplatin & $0.69[0.48-0.99]$ & \\
No & 1 & 0.98 \\
Nes & $1.26[0.81-1.98]$ & \\
No & $1.00[0.88-1.13]$ & \\
Yes & & \\
Number of LM & $\mathbf{3}$ & \\
No LM & $1.05[0.56-1.9]$ & \\
Yes & & \\
Major hepatectomy & $1.11[0.54-2.21]$ & \\
No & & \\
Yes & & \\
\hline
\end{tabular}

HIPEC: hyperthermic intraperitoneal chemotherapy; PCI: peritoneal cancer index; CCR: completeness of cytoreductive surgery, 0 : no visible residual disease; 1 : residual disease $\leq 2.5 \mathrm{~mm}$; 2 : residual disease $>2.5$ and $\leq 5 \mathrm{~mm}$; CCR 3 : residual disease $>5 \mathrm{~mm}$; EPIC: early postoperative intraperitoneal chemotherapy; CT: systemic chemotherapy; PM: Peritoneal metastases; LM: Liver metastases; Major hepatectomy was defined as the liver resection of at least three hepatic segments; R0 resection: margin width $>1 \mathrm{~mm}$; $\mathrm{R} 1$ resection: margin width $\leq 1 \mathrm{~mm}$; NS: Not significant. *Treatment after year 2000: the analysis was stratified by time periods (before and after 2000) to discern the effect of modern oxaliplatin-based chemotherapy. surgical sequencing of this select group of patients. However, this conclusion is in contrast with the results obtained with the only experimental model known to assess PM growth after LR [41]. Furthermore, the patients who had liver-first approach and then never went on to receive CRS and HIPEC, because of the progression, are not included in the analysis. Thus, the cohort of patients treated by liver-first approach and then by CRS and HIPEC represents a highly selected population of patients with better biology than those who never progress to the second step procedure. Limited peritoneal disease extent should be an advantage to explain better survival after liver first approach. Should be possible that the survival advantage was not related to the surgical sequence but to lower peritoneal tumor load. However, as shown in Table 2, the difference between PCI values in function of the surgical sequence is very low and does not explain the result. More investigations are necessary in order to conclude.

A limitation of this study is the period of data collection from numerous institutions during which temporal trends such as the advent of modern systemic chemotherapy has also taken place. To minimize this bias, the analysis was stratified by time periods (before and after 2000) to discern the effect of modern oxaliplatinbased. No impact in OS and RFS was observed in 56 (10\%) of patients were treated with extended surgical approach before 2003 . However, the small sample size could explain the non-significance of the result.

Despite the encouraging OS rates in our study, almost $50 \%$ of patients recurred within the first eighteen postoperative months. We also found an association between reduced RFS and severe complication events $(\mathrm{HR}=1.19$ [IC 95\% 0.98-1.44], $\mathrm{p}=0.081)$, which is in line with previous studies [42]. However, we also found that OS was surprisingly not related to severe morbidity as an earlier recurrence was assumed to be related to shorter survival. Varban et al. have also reported similar results [34]. Thus, these data suggest that careful selection of patients, less likely to experience severe postoperative complications, may allow for improved RFS. Nevertheless, the association of complications and survival should be attentively considered when selecting any patient with LM and PM for surgery. 
Table 6

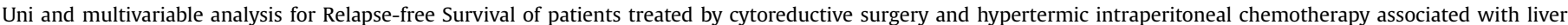
resection for liver and peritoneal metastases from colorectal cancer.

\begin{tabular}{|c|c|c|c|c|}
\hline & \multicolumn{2}{|l|}{ Univariate analysis } & \multicolumn{2}{|c|}{ Multivariate analysis } \\
\hline & $\operatorname{HR}[95 \% \mathrm{CI}]$ & $\mathrm{p}$ value & HR [95\% CI] & $\mathrm{p}$ value \\
\hline Age & $1[0.99-1.01]$ & 0.55 & & \\
\hline Age $>60$ years & & 0.29 & & \\
\hline No & 1 & & & \\
\hline Yes & $0.87[0.67-1.13]$ & & & \\
\hline Gender & & 0.84 & & \\
\hline Female & 1 & & & \\
\hline Male & $1.03[0.8-1.32]$ & & & \\
\hline Primary Tumor side & & 0.46 & & \\
\hline Right & 1 & & & \\
\hline Left & $0.81[0.62-1.05]$ & & & \\
\hline Rectum & $0.93[0.64-1.38]$ & & & \\
\hline Multifocal & $0.88[0.22-3.57]$ & & & \\
\hline Primary tumor Differentiation & & 0.49 & & \\
\hline Good & 1 & & & \\
\hline Moderate & $1.04[0.72-1.51]$ & & & \\
\hline Poor & $1.27[0.82-1.95]$ & & & \\
\hline T from the TNM & & 0.39 & & \\
\hline T3 & 1 & & & \\
\hline $\mathrm{T} 1$ or $\mathrm{T} 2$ & $1.69[0.78-3.66]$ & & & \\
\hline $\mathrm{T} 4$ & $1.07[0.79-1.44]$ & & & \\
\hline $\mathrm{N}$ from the TNM & & 0.1 & & 0.087 \\
\hline NO & 1 & & 1 & \\
\hline N1 & $1.33[0.9-1.96]$ & & $1.36[0.89-2.06]$ & \\
\hline N2N3 & $1.52[1.03-2.23]$ & & $1.57[1.04-2.37]$ & \\
\hline Treatment after year 2000 * & & 0.066 & & \\
\hline No & 1 & & & \\
\hline Yes & $0.69[0.47-1.03]$ & & & \\
\hline Surgical Sequences & & 0.036 & & NS \\
\hline One step & 1 & & & \\
\hline Liver first & $0.6[0.41-0.89]$ & & & \\
\hline HIPEC first & $0.89[0.55-1.42]$ & & & \\
\hline Sequence & & 0.02 & & NS \\
\hline One step & 1 & & & \\
\hline Two steps & $0.69[0.5-0.95]$ & & & \\
\hline PCI (for each more point) & $1.05[1.04-1.07]$ & $<0.0001$ & $1.07[1.05-1.09]$ & $<0.0001$ \\
\hline $\mathrm{PCI}>12$ & & $<0.0001$ & & NS \\
\hline No & 1 & & & \\
\hline Yes & $1.98[1.53-2.56]$ & & & \\
\hline CCR & & 0.66 & & \\
\hline 0 & 1 & & & \\
\hline 1 & $1.09[0.76-1.56]$ & & & \\
\hline EPIC & & 0.4 & & \\
\hline No & 1 & & & \\
\hline Yes & $1.21[0.77-1.91]$ & & & \\
\hline Intraperitoneal Oxaliplatin & & 0.07 & & NS \\
\hline No & 1 & & & \\
\hline Yes & $0.8[0.63-1.02]$ & & & \\
\hline Neoadjuvant CT for PM & & 0.79 & & \\
\hline No & 1 & & & \\
\hline Yes & $0.96[0.73-1.28]$ & & & \\
\hline Adjuvant CT for PM & & 0.5 & & \\
\hline No & 1 & & & \\
\hline Yes & $0.92[0.72-1.17]$ & & & \\
\hline Number of LM & $1.04[0.96-1.12]$ & 0.38 & & \\
\hline More than 3 LM & & 0.34 & & \\
\hline No & 1 & & & \\
\hline Yes & $1.21[0.81-1.81]$ & & & \\
\hline Major hepatectomy & & 0.65 & & \\
\hline No & 1 & & & \\
\hline Yes & $0.9[0.58-1.41]$ & & & \\
\hline Liver resection & & 0.47 & & \\
\hline R0 & 1 & & & \\
\hline R1 & $0.8[0.44-1.46]$ & & & \\
\hline Adjuvant $\mathrm{CT}$ after liver resection & & 0.0081 & & 0.15 \\
\hline No & 1 & & 1 & \\
\hline Yes & $0.71[0.55-0.92]$ & & $0.8[0.59-1.08]$ & \\
\hline
\end{tabular}

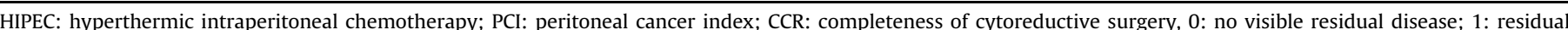

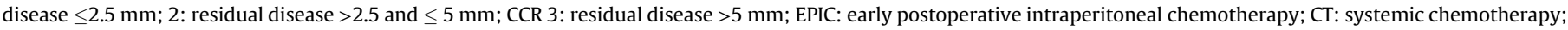

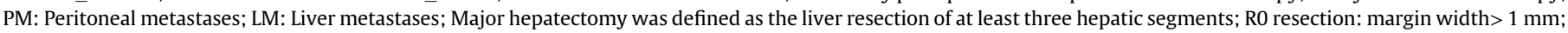

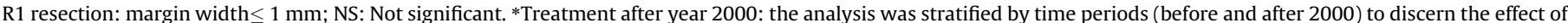
modern oxaliplatin-based chemotherapy. 
The promising long-term results of LM surgery from CRC over the past decade and recent trends towards increasing surgical aggressiveness (as illustrated by iterative resections of LM)[43] have formed the rationale for the surgical management of both LM and PM, given that CRS + HIPEC may also achieve excellent outcomes. Some series suggest that relatively long survival may be achieved with aggressive management, including the simultaneous resection of LM and PM [19,44,45]. Previous findings were confirmed in the present study showing that OS is significantly prolonged up to 60 months in selected cases. However, a metaanalysis of de Cuba et al. showed that patients with synchronous PM and LM of CRC seemed to fare less well when compared to patients with isolated PM (pooled HR $=1.24,95 \%$ CI $0.96-1.60)^{20}$. Despite this, the authors also showed a tendency towards better OS in carefully selected patients with PM and LM who were treated with curative resection of both sites plus HIPEC compared to treatment with modern systemic chemotherapy alone.

The PCI is considered the most widely used tool to evaluate disease extent in peritoneal surface malignancies [16,46-48]. Increased $\mathrm{PCI}$ is also recognized as an independent prognostic indicator for long-term outcomes in patients with PM from CRC [49] and an inverse linear relationship between any point rise in $\mathrm{PCI}$ and OS has been demonstrated [50]. Similarly, we found that PCI was an independent prognostic factor for OS and RFS in patients undergoing simultaneous resection of LM and PM. This result is in line with previous Italian two-center study reporting worse outcomes in patients with peritoneal than non-peritoneal colorectal metastases. However, survival benefit may be obtained in selected patients with limited peritoneal involvement [51]. Whilst PCI also influences the likelihood of complete cytoreduction [11,52], in the present analysis, the quality of CRS was optimal in close to $95 \%$ of the patients, with a reasonable mean PCI of about 10 . This finding is in line with the literature, as previous studies have shown that a large volume of disease was associated with poor long-term survival even if complete cytoreduction was achieved [47,49].

The concomitant presence of LM is considered a poor prognostic factor compared to patients with PM alone [16,20,45]. Elias et al. reported that completely resected LM during CRS remained a negative prognostic factor for patients with PM of CRC [53]. However, Maggiori et al. suggested that in LM and PM, prolonged survival may still be achieved in highly selected patients with limited peritoneal disease $(\mathrm{PCI}<12)$ [44]. In our study, we also found that a $\mathrm{PCl}>12$ was associated with a poor OS in both uni- and multivariate analysis. Therefore, the PCI itself could be a useful criterion for patient selection. In line with de Cuba et al., we also believe that, based on current data, there is no evidence to support an exclusion of patients with PM and LM from aggressive, potentially curative treatment [20]. However, an accurate, extensive preoperative evaluation is mandatory before surgery, and thus a diagnostic laparoscopy may prove useful in avoiding unnecessary surgery in high PCI and simultaneous CRC LM patients [54].

The recent results of the PRODIGE 7 [13], a prospective randomized multicenter phase III French trial, reopen the question concerning the benefit on survival outcomes given by the addition of HIPEC to the CRS compared to the CRS alone. Is it thus possible that CRS plus LR without HIPEC could have the same results of survival outcomes and lesser morbidity rates compared to the CRS plus LR with HIPEC? CRS plus LR without HIPEC has been reported in only rare and small series $[55,56]$. Allard et al. showed that combined resection of LM and limited peritoneal deposits (median PCI: 2) accidently discovered intra-operatively was associated with an $18 \%$ of 5-year OS rate and a median OS of 42 months [56]. However, in our study combined resection of LM and PM with HIPEC is associated with a better OS of 47 months with 5-year OS rates being $38 \%$ in patients with a higher PCI (median PCI: 10 ).
These better results are probably associated with a better selection of patients and the intrinsic properties of IP chemotherapy: the cytotoxic effects to the hyperthermia and the high local concentration compared to the systemic chemotherapy. Our conclusion is that the option of resection without HIPEC may potentially be proposed in cases where there is concern that HIPEC may increase in morbidity.

Currently, two regimens are widely used open-abdomen Oxaliplatin \pm Irinotecan with concurrent intravenous 5-fluorouracil and folinic acid, and open- or close-abdomen Mitomycin-C, alone or in combination with other drugs [12]. In this series we found that the use of Oxaliplatin vs other IP regimens had better outcomes. The improvement on the OS of the PM from CRC using modern Oxaliplatin-based chemotherapies is known. The role of the IP Oxaliplatine remains unclear. As previously reported most cytoreductive surgeons doing HIPEC with Oxaliplatin carried out the hyperthermic perfusion only for $30 \mathrm{~min}$, and most of those doing HIPEC with MMC did it for $90 \mathrm{~min}$. It is possible that the duration of hyperthermia, and not the drug itself could be responsible for survival difference [57]. However, in the same study patients considered with negative prognostic factors had better results in terms of survival if treated with IP Oxaliplatine. We considered that the presence of two metastatic sites, liver and peritoneum, in our population is a prognostic factor and this would explain the better result if treated with Oxaliplatin.

Despite analyzing prospectively maintained databases, this study is limited by its retrospective design resulting in some missing data. Furthermore, great heterogeneity in patient selection and operative techniques may have compromised our findings. However, despite these short-comings, this study represents the largest multicenter series, to date, and the data provided herein forms a basis for future prospective trials.

\section{Conclusion}

This multicenter study demonstrated the feasibility of the simultaneous treatment of colorectal cancer dissemination to the liver and peritoneum with liver resection, cytoreductive surgery and hyperthermic intraperitoneal chemotherapy in combination to systemic chemotherapy in selected patients, resulted in a 48month median overall survival, with reasonable morbidity. Even though this study suggests performing liver-first approach in the case of two-steps procedures, the exact timing of these individual complex treatment steps remains unknown. Future studies assessing the feasibility of this surgical approach in a prospective, randomized setting, as well as further studies elucidating how best to control disease progression following disease recurrence, would be helpful in improving the care of patients with advanced colorectal cancer.

\section{Sources of support}

No funding - No financial relationships.

\section{Author statement}

Study concepts: LO DICO Rea.

Study design: LO DICO Rea, POCARD Marc, MORRIS David, SAMMARTINO Paolo.

Data acquisition: LO DICO Rea.

Quality control of data and algorithms: LO DICO Rea, SAMMARTINO Paolo, POCARD Marc.

Data analysis and interpretation:all authors.

Statistical analysis: FARON Mathieu, LO DICO Rea.

Manuscript preparation: all authors. 
Manuscript editing: all authors.

Manuscript review: all authors.

\section{Declaration of competing interest}

None to declare.

\section{Acknowledgements}

The collaborators of the BIG-RENAPE Working Group are listed in Acknowledgements and include the following:

J. Abba (Department of Surgical Oncology, CHU Grenoble University, Grenoble, France); K. Abboud (Department of General Surgery, Centre Hospitalier Universitaire, Jean Monnet University, Saint Étienne, France); C. Arvieux (Department of Surgical Oncology, CHU Grenoble University, Grenoble, France); S. Carrere (Department of Surgical Oncology, Institut du Cancer de Montpellier, Montpellier, France); O. Facy (Department of Surgical Oncology, CHU Dijon, Dijon, France); G. Ferron (Department of Surgical Oncology, IUCT Oncopole, Toulouse, France); G. Lorimier (Department of Surgical Oncology, ICO-Paul Papin, Angers, France); F. Marchal (Department of Surgical Oncology, Institut de Cancerologie de Lorraine, Vandoeuvre-les-Nancy, France; P. OrtegaDeballon (Department of Surgical Oncology, CHU Dijon, Dijon, France); P. Rat (Department of Surgical Oncology, CHU Dijon, Dijon, France); J.-J. Tuech (Department of Digestive Surgery, Charles Nicolle University Hospital, Rouen, France), Laurent Villeneuve (Department of Surgical Oncology, Hospices Civils de Lyon).

\section{References}

[1] Hellman S, Weichselbaum RR. Oligometastases. J Clin Oncol. 1995 Jan;13(1): $8-10$.

[2] Nordlinger B, Sorbye H, Glimelius B, et al. Perioperative chemotherapy with FOLFOX4 and surgery versus surgery alone for resectable liver metastases from colorectal cancer (EORTC Intergroup trial 40983): a randomised controlled trial. Lancet 2008:371:1007-16.

[3] Koopman M, Antonini NF, Douma J, et al. Sequential versus combination chemotherapy with capecitabine, irinotecan, and oxaliplatin in advanced colorectal cancer (CAIRO): a phase III randomised controlled trial. Lancet 2007:370:135-42.

[4] Cremolini C, Loupakis F, Antoniotti C, et al. FOLFOXIRI plus bevacizumab versus FOLFIRI plus bevacizumab as first-line treatment of patients with metastatic colorectal cancer: updated overall survival and molecular subgroup analyses of the open-label, phase 3 TRIBE study. Lancet Oncol 2015;16: 1306-15.

[5] Franko J, Shi Q, Meyers JP, et al. Prognosis of patients with peritoneal metastatic colorectal cancer given systemic therapy: an analysis of individual patient data from prospective randomised trials from the Analysis and Research in Cancers of the Digestive System (ARCAD) database. Lancet Oncol 2016;17: 1709-19.

[6] Lemmens VE, Klaver YL, Verwaal VJ, et al. Predictors and survival of synchronous peritoneal carcinomatosis of colorectal origin: a population-based study. Int J Canc 2011;128:2717-25.

[7] Segelman J, Granath F, Holm T, et al. Incidence, prevalence and risk factors for peritoneal carcinomatosis from colorectal cancer. Br J Surg 2012;99:699-705.

[8] van Gestel YR, Thomassen I, Lemmens VE, et al. Metachronous peritonea carcinomatosis after curative treatment of colorectal cancer. Eur J Surg Onco 2014;40:963-9.

[9] Bengtsson G, Carlsson G, Hafstrom L, et al. Natural history of patients with untreated liver metastases from colorectal cancer. Am J Surg 1981:141:586-9.

[10] Koppe MJ, Boerman OC, Oyen WJ, et al. Peritoneal carcinomatosis of colorecta origin: incidence and current treatment strategies. Ann Surg 2006;243: $212-22$.

[11] Verwaal VJ, van Ruth S, de Bree E, et al. Randomized trial of cytoreduction and hyperthermic intraperitoneal chemotherapy versus systemic chemotherapy and palliative surgery in patients with peritoneal carcinomatosis of colorectal cancer. J Clin Oncol 2003;21:3737-43.

[12] Baratti D, Kusamura S, Pietrantonio F, et al. Progress in treatments for colorectal cancer peritoneal metastases during the years 2010-2015. A systematic review. Crit Rev Oncol Hematol 2016;100:209-22.

[13] Quenet F, Elias D, Roca L, et al. A UNICANCER phase III trial of hyperthermic intra-peritoneal chemotherapy (HIPEC) for colorectal peritoneal carcinomatosis (PC): PRODIGE 7. J Clin Oncol 2018;36. LBA3503-LBA3503.

[14] Tanaka T, Ozawa H, Nakagawa Y, Hirata A, Fujita S, Sugihara K. Verifying the
M1c category of CRC: analysis of the data from a Japanese multi-institutional database. Int J Colorectal Dis 2020 Jan;35(1):125-31.

[15] Fong Y, Fortner J, Sun RL, et al. Clinical score for predicting recurrence after hepatic resection for metastatic colorectal cancer: analysis of 1001 consecutive cases. Ann Surg 1999;230:309-18. discussion 318-21.

[16] Glehen O, Kwiatkowski F, Sugarbaker PH, et al. Cytoreductive surgery combined with perioperative intraperitoneal chemotherapy for the management of peritoneal carcinomatosis from colorectal cancer: a multi-institutional study. J Clin Oncol 2004:22:3284-92.

[17] Kianmanesh R, Scaringi S, Sabate JM, et al. Iterative cytoreductive surgery associated with hyperthermic intraperitoneal chemotherapy for treatment of peritoneal carcinomatosis of colorectal origin with or without liver metastases. Ann Surg 2007;245:597-603.

[18] Izzo F, Piccirillo M, Palaia R, et al. Management of colorectal liver metastases in patients with peritoneal carcinomatosis. J Surg Oncol 2009;100:345-7.

[19] Chua TC, Yan TD, Zhao J, et al. Peritoneal carcinomatosis and liver metastases from colorectal cancer treated with cytoreductive surgery perioperative intraperitoneal chemotherapy and liver resection. Eur J Surg Oncol 2009;35: $1299-305$.

[20] de Cuba EM, Kwakman R, Knol DL, et al. Cytoreductive surgery and HIPEC for peritoneal metastases combined with curative treatment of colorectal liver metastases: systematic review of all literature and meta-analysis of observational studies. Canc Treat Rev 2013;39:321-7.

[21] Berger Y, Aycart S, Tabrizian P, et al. Cytoreductive surgery and hyperthermic intraperitoneal chemotherapy in patients with liver involvement. J Surg Oncol 2016;113:432-7.

[22] Delhorme JB, Dupont-Kazma L, Addeo P, et al. Peritoneal carcinomatosis with synchronous liver metastases from colorectal cancer: who will benefit from complete cytoreductive surgery? Int J Surg 2016;25:98-105.

[23] Navez J, Remue C, Leonard D, et al. Surgical treatment of colorectal cancer with peritoneal and liver metastases using combined liver and cytoreductive surgery and hyperthermic intraperitoneal chemotherapy: report from a single-centre experience. Ann Surg Oncol 2016;23:666-73.

[24] Lorimier G, Linot B, Paillocher N, et al. Curative cytoreductive surgery followed by hyperthermic intraperitoneal chemotherapy in patients with peritoneal carcinomatosis and synchronous resectable liver metastases arising from colorectal cancer. Eur J Surg Oncol 2017;43:150-8.

[25] Pinto A, Hobeika C, Philis A, et al. Synchronous liver metastases and peritoneal carcinomatosis from colorectal cancer: different strategies for curative treatment? Langenbeck's Arch Surg 2019;404:477-88.

[26] Dindo D, Demartines N, Clavien PA. Classification of surgical complications: a new proposal with evaluation in a cohort of 6336 patients and results of a survey. Ann Surg 2004;240:205-13.

[27] Jacquet P, Sugarbaker PH. Clinical research methodologies in diagnosis and staging of patients with peritoneal carcinomatosis. Canc Treat Res 1996;82: 359-74.

[28] Basch E, Reeve BB, Mitchell SA, et al. Development of the National Cancer Institute's patient-reported outcomes version of the common terminology criteria for adverse events (PRO-CTCAE). J Natl Cancer Inst 2014;106.

[29] Evers DJ, Verwaal VJ. Indication for oophorectomy during cytoreduction for intraperitoneal metastatic spread of colorectal or appendiceal origin. Br J Surg 2011;98:287-92.

[30] Brigand C, Monneuse O, Mohamed F, et al. Peritoneal mesothelioma treated by cytoreductive surgery and intraperitoneal hyperthermic chemotherapy: results of a prospective study. Ann Surg Oncol 2006;13:405-12.

[31] Elias D, Goere D, Dumont F, et al. Role of hyperthermic intraoperative peritoneal chemotherapy in the management of peritoneal metastases. Eur J Canc 2014;50:332-40.

[32] Schemper M, Smith T. A note of quantifying follow-up in studies of failure time. Contr Clin Trials 1999;17:343-6.

[33] Thomassen I, van Gestel YR, Lemmens VE, et al. Incidence, prognosis, and treatment options for patients with synchronous peritoneal carcinomatosis and liver metastases from colorectal origin. Dis Colon Rectum 2013;56: $1373-80$.

[34] Varban O, Levine EA, Stewart JH, et al. Outcomes associated with cytoreductive surgery and intraperitoneal hyperthermic chemotherapy in colorectal cancer patients with peritoneal surface disease and hepatic metastases. Cancer 2009;115:3427-36.

[35] Loupakis F, Cremolini C, Masi G, et al. Initial therapy with FOLFOXIRI and bevacizumab for metastatic colorectal cancer. N Engl J Med 2014;371: $1609-18$.

[36] Imamura H, Seyama Y, Kokudo N, et al. One thousand fifty-six hepatectomies without mortality in 8 years. Arch Surg 2003;138:1198-206. discussion 1206.

[37] Jarnagin WR, Gonen M, Fong Y, et al: Improvement in perioperative outcome after hepatic resection: analysis of 1,803 consecutive cases over the past decade. Ann Surg 236:397-406; discussion 406-7, 2002.

[38] Sano T, Shimada K, Sakamoto Y, et al. One hundred two consecutive hepatobiliary resections for perihilar cholangiocarcinoma with zero mortality. Ann Surg 2006;244:240-7.

[39] Adam R, de Gramont A, Figueras J, et al. Managing synchronous liver metastases from colorectal cancer: a multidisciplinary international consensus. Canc Treat Rev 2015;41:729-41.

[40] Gavriilidis P, Katsanos K, Sutcliffe RP, et al. Simultaneous, delayed and liverfirst hepatic resections for synchronous colorectal liver metastases: a systematic review and network meta-analysis. J Clin Med Res 2019;11:572-82. 
[41] Lo Dico R, Tijeras-Raballand A, Bonnin P, et al. Hepatectomy increases metastatic graft and growth in an immunocompetent murine model of peritoneal metastases. Eur J Surg Oncol 2018;44:784-91.

[42] Simkens GA, van Oudheusden TR, Luyer MD, et al. Serious postoperative complications affect early recurrence after cytoreductive surgery and HIPEC for colorectal peritoneal carcinomatosis. Ann Surg Oncol 2015;22:2656-62.

[43] Taylor A Kg. Survival after surgical resection of hepatic metastases from colorectal cancer: a systematic review and meta-analysis. Ann Oncol 2010;21(8:632).

[44] Maggiori L, Goere D, Viana B, et al. Should patients with peritoneal carcinomatosis of colorectal origin with synchronous liver metastases be treated with a curative intent? A case-control study. Ann Surg 2013;258:116-21.

[45] Elias D, Faron M, Goere D, et al. A simple tumor load-based nomogram for surgery in patients with colorectal liver and peritoneal metastases. Ann Surg Oncol 2014;21:2052-8.

[46] Koh JL, Yan TD, Glenn D, et al. Evaluation of preoperative computed tomography in estimating peritoneal cancer index in colorectal peritoneal carcinomatosis. Ann Surg Oncol 2009;16:327-33.

[47] Pestieau SR, Sugarbaker PH. Treatment of primary colon cancer with peritoneal carcinomatosis: comparison of concomitant vs. delayed management. Dis Colon Rectum 2000;43:1341-6. discussion 1347-8.

[48] Shehata M, Chu F, Saunders V, et al. Peritoneal carcinomatosis from colorectal cancer and small bowel cancer treated with peritonectomy. ANZ J Surg 2006:76:467-71.

[49] da Silva RG, Sugarbaker PH. Analysis of prognostic factors in seventy patients having a complete cytoreduction plus perioperative intraperitoneal chemotherapy for carcinomatosis from colorectal cancer. J Am Coll Surg 2006;203: 878-86.

[50] Faron M, Macovei R, Goere D, et al. Linear relationship of peritoneal cancer index and survival in patients with peritoneal metastases from colorecta cancer. Ann Surg Oncol 2016;23:114-9.

[51] Baratti D, Kusamura S, Iusco D, et al. Should a history of extraperitoneal disease Be a contraindication to cytoreductive surgery and hyperthermic intraperitoneal chemotherapy for colorectal cancer peritoneal metastases? Dis Colon Rectum 2018;61:1026-34.

[52] Yan TD, Chu F, Links M, et al. Cytoreductive surgery and perioperative intraperitoneal chemotherapy for peritoneal carcinomatosis from colorectal carcinoma: non-mucinous tumour associated with an improved survival. Eur ] Surg Oncol 2006;32:1119-24.

[53] Elias D, Gilly F, Boutitie F, et al. Peritoneal colorectal carcinomatosis treated with surgery and perioperative intraperitoneal chemotherapy: retrospective analysis of 523 patients from a multicentric French study. J Clin Oncol 2010;28:63-8.

[54] Najah H, Lo Dico R, Dohan A, et al. A feasibility study of the use of computed virtual chromoendoscopy for laparoscopic evaluation of peritoneal metastases. Surg Endosc 2017;31:743-51.

[55] Desolneux GMaziere C, Vara J. Cytoreductive surgery of colorectal peritonea metastases: outcomes after complete cytoreductive surgery and systemic chemotherapy only. PLoS One 2015 Mar 31;10(3):e0122816.

[56] Allard MA, Adam R, Ruiz A, et al. Is unexpected peritoneal carcinomatosis stil a contraindication for resection of colorectal liver metastases? Combined resection of colorectal liver metastases with peritoneal deposits discovered intra-operatively. EJSO 2013;39. 981e987.

[57] Prada-Villaverde A, Esquivel J, Lowy AM, et al. The American Society of Peritoneal Surface Malignancies evaluation of HIPEC with Mitomycin C versus Oxaliplatin in 539 patients with colon cancer undergoing a complete cytoreductive surgery. J Surg Oncol 2014 Dec;110(7):779-85. 